\title{
Effects of surface cracks and strain rate on the tensile behavior of Balmoral Red granite
}

\author{
Ahmad Mardoukhi ${ }^{a}$, Mikko Hokka, and Veli-Tapani Kuokkala \\ Tampere University of Technology, Department of Material Science, POB 589, 33101 Tampere, Finland
}

\begin{abstract}
This paper presents an experimental procedure for studying the effects of surface cracks on the mechanical behavior of Balmoral Red granite under dynamic and quasi-static loading. Three different thermal shocks were applied on the surface of the Brazilian Disc test samples by keeping a flame torch at a fixed distance from the sample surface for 10, 30, and 60 seconds. Microscopy clearly shows that the number of the surface cracks increases with the duration of the thermal shock. After the thermal shock, the Brazilian Disc tests were performed using a servohydraulic materials testing machine and a compression Split Hopkinson Pressure Bar (SHPB) device. The results show that the tensile strength of the rock decreases and the rate sensitivity of the rock increases as more cracks are introduced to the structure. The DIC analysis of the Brazilian disc tests shows that the fracture of the sample initiates at the center of the samples or slightly closer to the incident bar contact point. This is followed by crushing of the samples at both contact points with the stress bars.
\end{abstract}

\section{Introduction}

Geothermal energy has recently attracted a lot of attention among engineers and scientists. However, there are still significant technological and scientific challenges to deal with before the lucrative energy resources can be easily reached. One of the biggest challenges is the drilling of the wells as deep as five kilometers to reach the required temperature to produce electricity. This challenge becomes bolder in the areas far away from the tectonic plate boundaries and in regions where the bedrock consists of hard rocks such as granite typically found in the Nordic countries. The current drilling technologies are based on rotary and percussive drilling. To improve the current drilling technology, the engineers need to know the rock strength and other properties at the drilling conditions, which include high hydrostatic pressures and elevated temperatures. However, since the straightforward experimental testing at these conditions is scientifically and practically very difficult, various numerical simulations and constitutive modeling approaches are currently being used for predicting the fracture behavior and the rock-tool interactions such as tool wear. Saksala et al. [6] studied the fracture behavior of Kuru granite by analyzing the Brazilian Disc tests with numerical and experimental approaches. In a separate study, Saksala et al. [7] extended the modeling approach to $3 \mathrm{D}$ and studied the bit-rock interactions and rock fracture in percussive drilling. Tang et al. [8,9] carried out numerical simulations to study the influence of microstructure on rock failure in uniaxial compression. They analyzed the effect of the structure heterogeneity of the rock on the fracture behavior and reported that the macro-fracture occurs abruptly as soon as the specimen

\footnotetext{
${ }^{a}$ Corresponding author: ahmad.mardoukhi@tut.fi
}

reaches the peak stress. They also reported observations of micro-cracks over the whole volume of the specimens prior to the macro-fracture, and that the splitting failure is the dominant mechanism in uniaxial loading. However, due to the heterogeneous nature of rocks, other kinds of failure mechanisms were also observed.

The construction of material models and validation of the simulation results, however, relies on good scientific understanding of the material behavior as well as experimental data for calibrating the material models. The dynamic testing of rocks, however, is challenging, especially with the bar methods, since the material fails at very low strains. Therefore, it is very difficult to achieve the stress equilibrium, and cracks tend to initiate on the incident loading side of the sample before the other parts of the sample are fully loaded. Luckily, noncontact full-field deformation analysis techniques, such as photoelasticity, Moire, coherent gradient sensor, and digital image correlation (DIC) can be used to analyze the fracture behavior during a dynamic impact [1-5].

From the scientific point of view, there are still various problems in the understanding of rock behavior. For instance, the crack initiation, propagation, and coalescence are not completely understood, and many observations cannot be explained by any solid theory. Therefore, a material model that takes into account these factors and predicts the material behavior is needed for high quality drilling simulations. For example, the simulations of several repeated impacts of the drill on the same location of the rock surface require understanding of the effects of microcracks formed during the first impact. However, producing reliable experimental data that accurately describes the rock behavior at the impact speeds is not an easy task. Percussive drilling occurs at impact speeds of around $5-20 \mathrm{~m} / \mathrm{s}$, and each impact causes dynamic loading on the rock. Therefore, understanding of the 

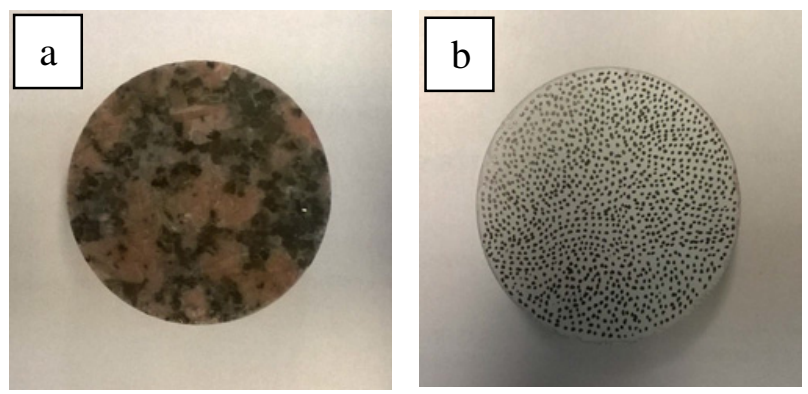

Figure 1. a) Natural surface of a Balmoral Red granite sample and b) DIC spackle pattern on the sample.

dynamic material behavior and obtaining numerical data are crucially important for the simulation purposes and material modeling, but also for the designing of new and more efficient drills and methods for faster and easier drilling. In this paper, the effects of microstructure, and especially the influence of surface cracks and the crack patterns on the mechanical behavior of the rock material were studied. The mechanical behavior of Balmoral Red granite was studied using Brazilian Disc (BD) tests at a wide range of strain rates. High speed photography and DIC were used to analyze the fracture behavior of the rock samples. The experimental setup, data acquisition, and interpretation of the results are presented in details.

\section{Experimental}

The material studied in this work was coarse grained Balmoral Red granite. This granite comprises Potash Feldspar, Quartz, Plagioclase, Biotite, and Hornblende. The mean value of the open porosity is $0.38 \%$ and the quasi-static compression strength is close to $180 \mathrm{MPa}$. The rock microstructure has no texture, the minerals are distributed homogeneously, and the mechanical properties are essentially isotropic. The Brazilian Disc samples with a diameter of $40.5 \mathrm{~mm}$ were cut from granite plates with a thickness of $21 \mathrm{~mm}$. Figure 1a shows the natural surface of the BD sample, and Fig. 1b the sample with the DIC spackle pattern. The samples were tested at different strain rates, and the effect of surface cracks was studied by applying different heat shocks to the samples. Before the thermal shocks, liquid penetrant and optical microscopy were used to analyze the pattern of the surface cracks. Thermal shocks of 10,30, and 60 seconds were applied on the BD samples by keeping the samples at a fixed distance from an oxygen-acetylene flame torch. After the heat shock, the samples were allowed to cool down to room temperature in air. After the cooling the liquid penetrant was re-applied on the surface to see the changes in the crack patterns caused by the thermal shock. The images were obtained using a stereomicroscope using UV light. Fractal dimensions of the surface crack patterns were calculated from the obtained images using a box counting method in Matlab.

The low strain tests were performed using an Instron 8800 servohydraulic materials testing machine with a displacement rate of $1 \mathrm{~mm} / \mathrm{s}$, and the high strain rate tests with a compression Split Hopkinson Pressure Bar

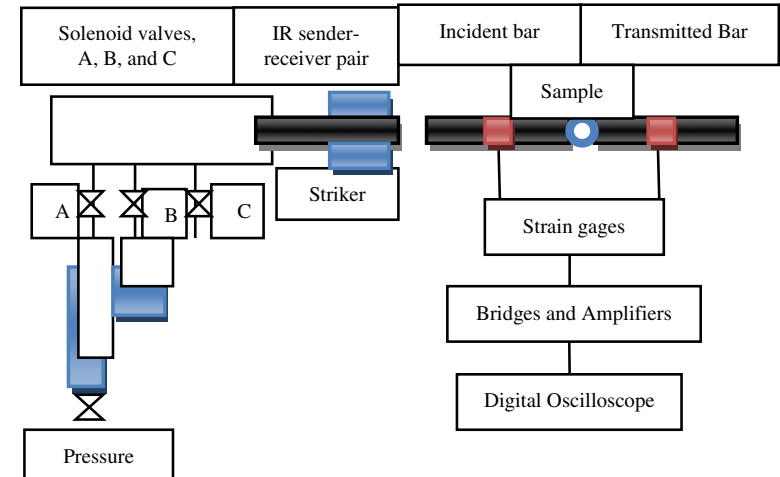

Figure 2. Schematic picture of the Split Hopkinson Pressure Bar device at Tampere University of Technology.

(SHPB). The pressure bars were of Maraging steel with a diameter of $22 \mathrm{~mm}$ and a length of $1200 \mathrm{~mm}$. The tests were performed using a $300 \mathrm{~mm}$ striker bar with an impact speed of $10 \mathrm{~m} / \mathrm{s}$. Each test was repeated five times. Two Photron SA-X2 high speed cameras were used to record the deformation and fracture of the samples during the high strain rate tests. As the rock surface itself does not provide a very strong contrast for the correlation algorithm, the surfaces of the samples were painted with white base coat and the black speckles were applied on it using a permanent ink marker, as shown in Fig. 1b. The images were recorded at $160 \mathrm{kfps}$, the size of the image at this rate being $256 * 176$ pixels. Two Decocool lights were used for illuminating the sample. The recorded images were analyzed using the LaVision StrainMaster (DaVis) 3D-DIC software. The displacements were calculated by comparing the deformed images to the first (reference) image using a step size of 9 pixels and a subset size of 25 pixels. The analyzed area was masked from the left reference image using a circular mask to cut out the outermost edges of the sample. The seed points were manually selected from areas that did not move much during the test. Figure 2 shows a schematic picture of the high strain rate testing setup.

The tensile stress in the center of the sample can be calculated when the force acting on the specimen is known. In this work, the forces on the sample surfaces were calculated from the incident and reflected pulses, and from the transmitted pulse. The forces on the incident and transmitted sides of the sample were compared with each other to verify stress equilibrium. However, the transmitted pulse typically contains fewer oscillations, and therefore it was used for calculating the tensile stress in the sample. The mathematical description for calculating the tensile stress from the contact forces is presented for example in ref. [6].

\section{Results and discussion}

Figure 3 shows an example of the optical analysis used to characterize the crack patterns on the sample surface. Figures $3 \mathrm{a}$ and $3 \mathrm{c}$ are the original grayscale optical images obtained from the sample before and after a 30 second heat shock, and Figs. $3 \mathrm{~b}$ and $3 \mathrm{~d}$ show the black and 


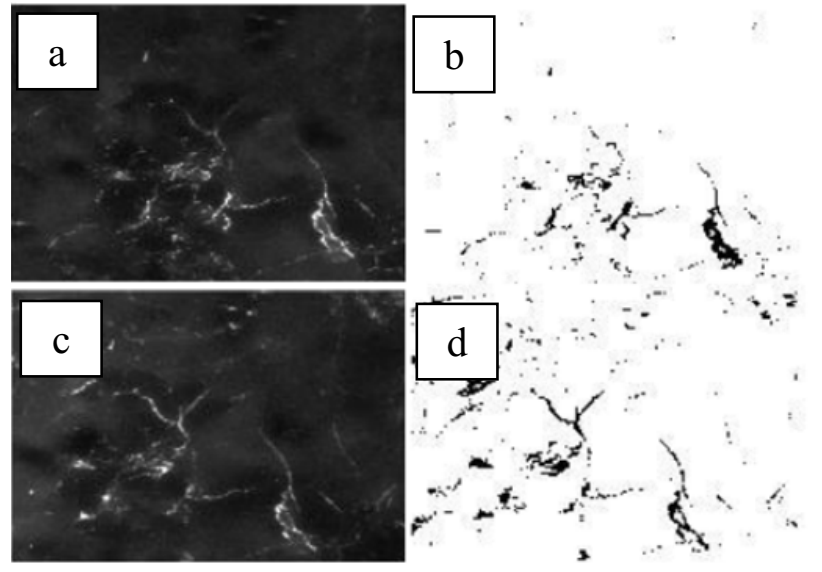

Figure 3. Optical images a) before and c) after the thermal shock, and identified cracks b) before and d) after the thermal shock.

Table 1. Fractal dimensions for the heat shocked samples.

\begin{tabular}{|l|l|l|l|l|l|l|}
\hline & \multicolumn{2}{l|}{ 10 Seconds } & \multicolumn{2}{l|}{ 30 Seconds } & \multicolumn{2}{l|}{ 60 Seconds } \\
\hline & Before & After & Before & After & Before & After \\
\hline & 1.3097 & 1.2583 & 1.1796 & 1.3631 & 1.4464 & 1.5132 \\
\hline & 1.2779 & 1.349 & 1.2498 & 1.2783 & 0.9976 & 1.4426 \\
\hline & 1.3254 & 1.3313 & 1.3819 & 1.4733 & 1.3375 & 1.4001 \\
\hline & 1.3192 & 1.3094 & 1.3567 & 1.4296 & 1.4912 & 1.5821 \\
\hline & 1.3239 & 1.324 & 1.4424 & 1.6094 & 1.518 & 1.5241 \\
\hline Average & $\mathbf{1 . 3 1 1 2 2}$ & $\mathbf{1 . 3 1 4 4}$ & $\mathbf{1 . 3 4 0 6}$ & $\mathbf{1 . 4 0 6}$ & $\mathbf{1 . 3 9 0}$ & $\mathbf{1 . 4 0 5}$ \\
\hline STD & $\mathbf{0 . 2 4 5}$ & $\mathbf{0 . 0 3 4 5}$ & $\mathbf{0 . 1 0 6}$ & $\mathbf{0 . 1 2 4}$ & $\mathbf{0 . 2 1 3}$ & $\mathbf{0 . 0 9 3 8}$ \\
\hline Change & & $\mathbf{0 . 3 \%}$ & & $\mathbf{8 \%}$ & & $\mathbf{8 \%}$ \\
\hline
\end{tabular}

white images after thresholding of the images shown in Figs. $3 \mathrm{a}$ and $\mathrm{c}$. The fractal dimensions of the crack patterns were calculated from the black and white images. The fractal dimensions of the crack patterns increase during the thermal shock, indicating that the pattern of the cracks after the thermal shock is more complex and the network contains more interconnected surface cracks.

The fractal dimensions were obtained for a total of 15 samples, i.e., five samples for each heat shock of 10, 30, and 60 seconds. The average of the fractal dimensions prior to the heat shock for all 15 samples was $1.334 \pm 0.13$. The effect of the heat shock on the crack pattern can be estimated from the change in the fractal dimensions. After the 10 second heat shock, the fractal dimensions are essentially the same as before the heat shock. However, after the 30 and 60 second heat shocks the fractal dimensions increase by $8 \%$. Unfortunately, there was a significant scatter in the fractal dimensions obtained prior to the heat shocks, and therefore the comparison of the effect of different heat shocks on the mechanical properties is somewhat limited. The relative change in the fractal dimensions, however, seems to correlate quite well with the change in the mechanical properties. Table 1 summarizes the fractal dimensions for the heat shocked samples before and after the heat shock.

The results of the low strain rate tests are shown in Fig. 4. The stress as a function of time for the granite specimens without any heat shocks are shown in Fig. 4a,

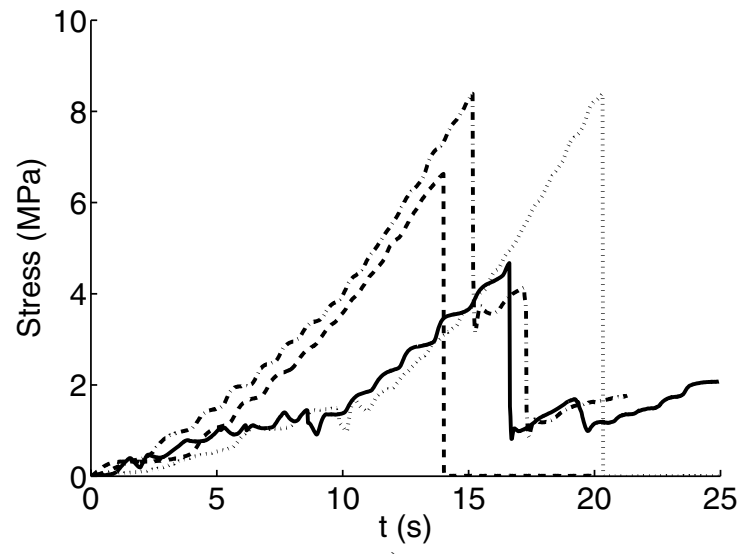

a)

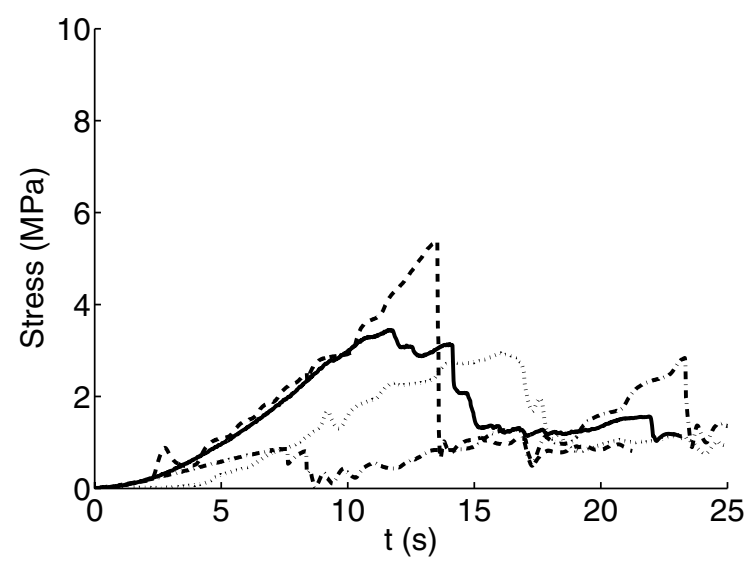

b)

Figure 4. Low strain rate test results showing the tensile stress as a function of time for a) samples without a thermal shock and b) samples with a 60 second thermal shock.

while Fig. $4 \mathrm{~b}$ shows the corresponding data for the samples with a 60 second heat shock. The tensile strength of the rock without a heat shock varies between $8 \mathrm{MPa}$ and $4 \mathrm{MPa}$, and the fracture of the sample always occurs as a very clear and rapid drop of the stress to essentially zero. However, after the 60 second heat shock, siginificant scatter appears in the fracture behavior of the rock, as can be seen in Fig. 4b. The heat shocked samples do not fail by only one rapidly propagating fracture, as observed for the rock samples without a heat shock (Fig. 4a), but the failure occurs by several smaller consecutive drops in the tensile stress. The strength of the rock also drops by about $50 \%$, with only one sample showing a significantly higher strenght. This behavior is evidently due to the increase in the number of cracks generated during the heat shocks, which facilitates the fracture and realease of the energy absorbed by the sample during the impact.

During the dynamic loading, the fracture behavior of the studied material is different and the scatter is significantly reduced. Figure 5 shows the tensile stress as a function of time for the samples without a heat shock (5a) and for the samples with a 60 second shock (5b). The average tensile strength obtained from the five dynamic tests on the non-shocked specimens is $28 \mathrm{MPa}$. The heat shock, however, decreases the dynamic strength significantly, as the average tensile strengths for the heat 


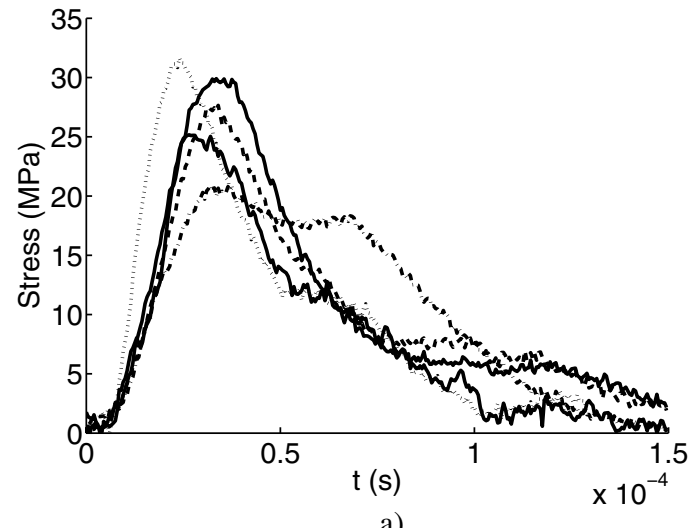

a)

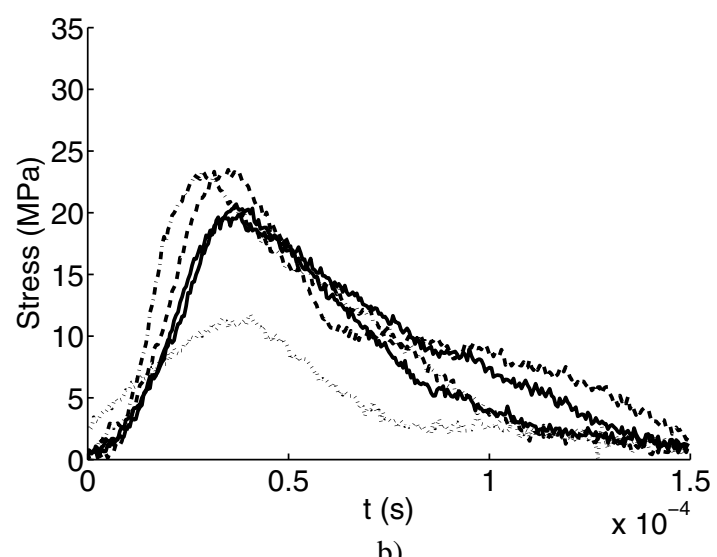

Figure 5. High strain rate test results showing the tensile stress as a function of time for samples a) without a heat shock and b) samples with a 60 second thermal shock.

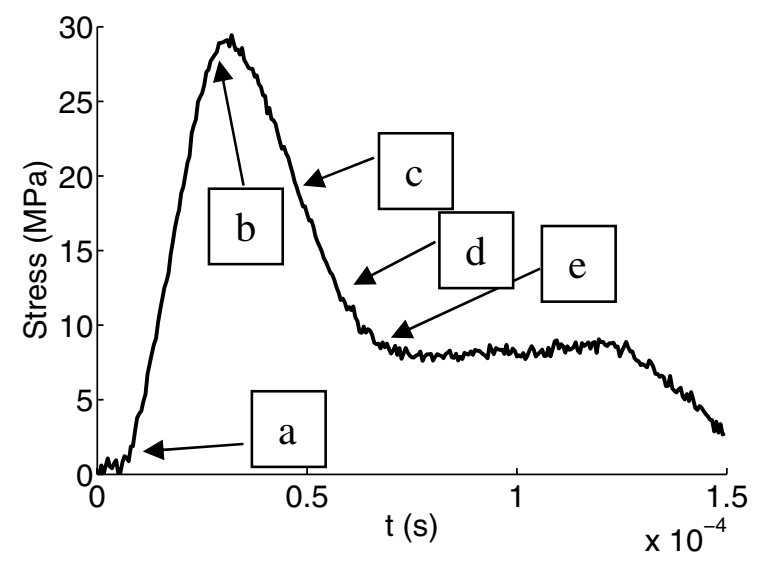

Figure 6. Tensile stress as a function of time for a sample with a 10 second thermal shock.

shocked samples are $23 \mathrm{MPa}, 22 \mathrm{MPa}, 19 \mathrm{MPa}$ for the heat shocks of 10, 30, and 60 seconds. As described before, the fractal dimensions of the surface cracks increase with the duration of the heat shock. This can be interpreted as an increase in the number of cracks on the surface, and more importantly as an increase of the complexity and interconnectivity of the crack network. Based on the mechanical testing, the increase in the fractal dimensions correlates with the duration of the heat shock and also with the decrease in the strength of the tested samples.
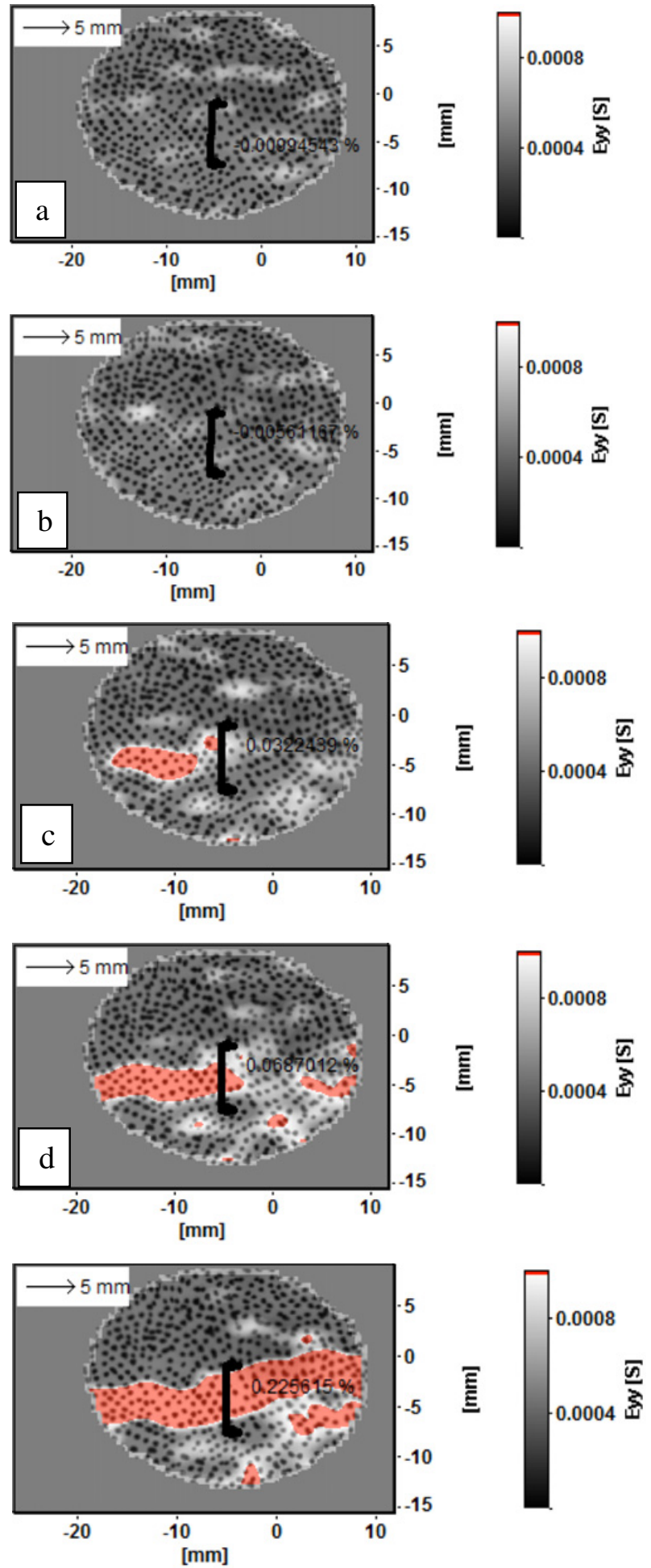

Figure 7. DIC images obtained during the dynamic loading of the sample for which the force-time plot is shown in Fig. 6. a) $\mathrm{t}=0 \mu \mathrm{s}, \mathrm{b}) \mathrm{t}=31.25 \mu \mathrm{s}, \mathrm{c}) \mathrm{t}=50 \mu \mathrm{s}, \mathrm{d}) \mathrm{t}=56.25 \mu \mathrm{s}$, and e) $\mathrm{t}=62.5 \mu \mathrm{s}$.

The 60 second heat shock decreases the low strain rate strength of the rock from $7 \pm 0.43 \mathrm{MPa}$ for the samples without a heat shock to $3.5 \pm 2.5 \mathrm{MPa}$ for the samples tested after the heat shock. The decrease of strength is therefore about $48 \%$. When the loading rate is increased, the same 60 second heat shock decreases the rock strength 
from $28 \pm 3.35 \mathrm{MPa}$ to $19 \pm 0.53 \mathrm{MPa}$, i.e., by $32 \%$. The increased loading rate therefore increases the rock strength by about $400 \%$ for the samples tested without a heat shock and by about $550 \%$ for the samples tested after a 60 second heat shock. Therefore, the relative increase in strength due to the rate effect seems to be stronger for the samples with a higher content of surface cracks.

The DIC analysis of the BD samples shows that the fracture of the specimens initiates at the center or slightly closer to the incident bar contact point. This observation and comparison of the forces on the incident and transmitted sides of the sample indicate adequate stress equilibrium. The engineering strain in the direction perpendicular to the loading direction prior to fracture was measured by placing a virtual strain gage at the center of the specimen using LaVision StrainMaster (DaVis) 3DDIC software. The results reveal a decreasing trend of failure strain as the duration of the heat shock increases. For the non-heat treated samples the maximum strain prior to failure is $0.8 \%$, while for the heat shocked samples the corresponding numbers are $0.6 \%, 0.6 \%$, and $0.5 \%$ for the 10,30 , and 60 second heat shocks. The relative decrease in the maximum strain before fracture seems to be quite similar to that observed for the decrease in strength.

Figure 6 shows the tensile stress as a function of time for a sample tested after a 10 second heat shock. Point (a) in the Figure corresponds to the start of the loading and (b) indicates the moment just before the maximum stress. The high speed images corresponding to the time stamps indicated in Fig. 6 are shown in Fig. 7. Also the strain maps in the Y-direction (vertical direction, perpendicular to loading) are overlaid with the original high speed images. It can be seen from the images that the strains in the two first images (7a and b) are very low and essentially constant over the sample surface. The first strain localization does not yet show in the image obtained just microseconds before the strength of the rock starts to decrease. This indicates that the fracture initiates inside the sample and does not appear on the surface at this point. As the fracture propagates, it becomes visible also in the strain maps (Fig. 7c) as a strong concentration of deformation. The crack then propagates through the sample very quickly in Figs. 7d and e. This is followed by crushing of the contact points of the sample with the stress bars.

\section{Conclusions}

The mechanical behavior of Balmoral Red granite was studied using the Brazilian disc tests at quasi-static and dynamic strain rates. The effect of surface cracks on the mechanical properties was studied by generating Brazilian disc samples with different surface crack patterns by modifying the rock surface structure using thermal shock treatments. The optical images and fractal dimensions obtained from the surface of the samples before and after the heat shock clearly show an increase in the amount of surface cracks and in the complexity of the crack network.

At quasi-static strain rates the fracture of the rock samples without a heat shock occurs as a sharp drop of strength at maximum stress. The samples tested after the heat shock show significant scatter in the strength and the final fracture can occur in several short steps instead of one clear drop. However, at high strain rates the rock shows more consistent behavior and the scatter is significantly reduced. Based on the results, the strain rate effect is stronger for the samples with a 60 second heat shock than for the samples without any heat shock.

Digital image correlation was used to observe the crack initiation and propagation on the surface of the samples during the high rate tests. Time stamps from the test were recorded on the images, and the data from the HSPB was synchronized with the obtained images. The cracks initiate typically inside the sample and propagate to the surface only after the strength of the sample has already decreased significantly. The DIC can also be used to estimate the fracture strain of the material during a high strain rate test. This can be done, for example, with a virtual extensometer placed on the image. The obtained maximum strains prior to the fracture also decrease with the heat shock duration.

This work was supported by the Finnish Research Foundation for Natural Resources under the grant no. 1768/14.

\section{References}

[1] W.H. Peters, W.F. Ranson, Opt. Eng. 21, 427 (1982)

[2] J.E. Field, S.M. Walley, W.G. Proud, H.T. 2. Goldrein, C.R. Siviour, Impact. Eng. 30, 725 (2004)

[3] F. Hild, S. Roux, Exp. Mech. 42, 69 (2006)

[4] V. Tiwari, M.A. Sutton, S.R. McNeill, Exp. Mech. 47, 561 (2007)

[5] P.L. Reu, T.J. Miller, Strain Anal. Eng. Des. 43, 673 (2008)

[6] T. Saksala, M. Hokka, V.T. Kuokkala, J. Mäkinen, Rock Mech. Mining Sci. 59, 128 (2013)

[7] T. Saksala, Numer. Anal. Met. 37, 309 (2012)

[8] C.A. Tang, H. Liu, P.K.K. Lee, Y. Tsui, L.G. 8. 8. Tham, Rock Mech. Mining Sci. 37, 555 (2000)

[9] C.A. Tang, L.G. Tham, P.K.K. Lee, Y. Tsui, H. Liu, Rock Mech. Mining Sci. 37, 571 (2000) 